\title{
Symptomatic treatments for amyotrophic lateral sclerosis/motor neuron disease (Protocol)
}

\author{
Ng L, Khan F, Young CA
}

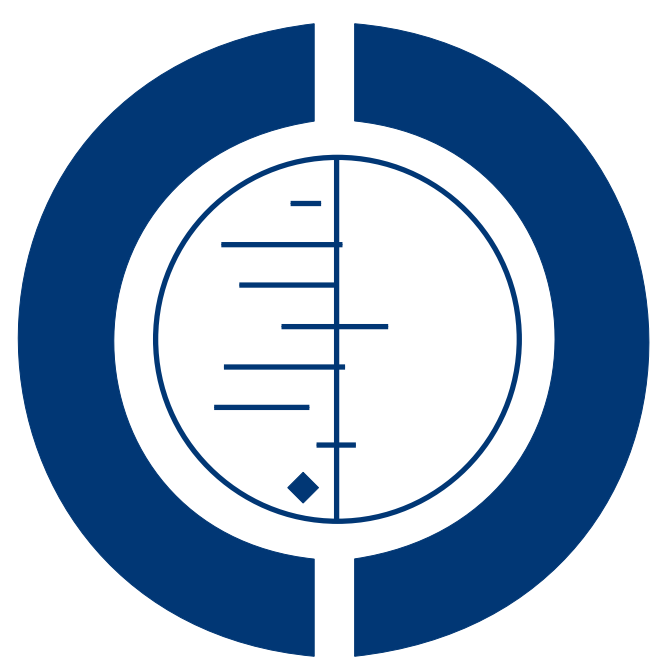

\section{THE COCHRANE COLLABORATION $^{\circledR}$}

This is a reprint of a Cochrane protocol, prepared and maintained by The Cochrane Collaboration and published in The Cochrane Library 2015, Issue 6

http://www.thecochranelibrary.com

\section{WILEY}


TABLE OF CONTENTS

HEADER . . . . . . . . . . . . . . . . . . . . . . . . . . . . . . . . . . . . 1

ABSTRACT . . . . . . . . . . . . . . . . . . . . . . . . . . . . . . . . . . . . . . . 1

BACKGROUND . . . . . . . . . . . . . . . . . . . . . . . . . . . . . . . . . . . .

OBJECTIVES . . . . . . . . . . . . . . . . . . . . . . . . . . . . . . . . . . . . . . .

METHODS . . . . . . . . . . . . . . . . . . . . . . . . . . . . . . . . . . . . . .

ACKNOWLEDGEMENTS . . . . . . . . . . . . . . . . . . . . . . . . . . . . . . . . . . . . . . .

REFERENCES . . . . . . . . . . . . . . . . . . . . . . . . . . . . . . . . . . . . . . . 5

APPENDICES . . . . . . . . . . . . . . . . . . . . . . . . . . . . . . . . . . . . . 7

CONTRIBUTIONS OF AUTHORS . . . . . . . . . . . . . . . . . . . . . . . . . . . . . . . . . . . . . . . . . . . . .

DECLARATIONS OF INTEREST . . . . . . . . . . . . . . . . . . . . . . . . . . . . . . . . . . 8

SOURCES OF SUPPORT . . . . . . . . . . . . . . . . . . . . . . . . . . . . . . . . . . . . . . . . . . . 
[Overview of Reviews Protocol]

\title{
Symptomatic treatments for amyotrophic lateral sclerosis/motor neuron disease
}

\author{
Louisa $\mathrm{Ng}^{1}$, Fary $\mathrm{Khan}^{1}$, Carolyn A Young ${ }^{2}$ \\ ${ }^{1}$ Department of Rehabilitation Medicine, Royal Melbourne Hospital, Royal Park Campus, Melbourne, Australia. ${ }^{2}$ The Walton Centre \\ NHS Foundation Trust, Liverpool, UK \\ Contact address: Louisa Ng, Department of Rehabilitation Medicine, Royal Melbourne Hospital, Royal Park Campus, Poplar Road, \\ Parkville, Melbourne, Victoria, 3052, Australia. louisa.ng@mh.org.au.
}

Editorial group: Cochrane Neuromuscular Disease Group.

Publication status and date: New, published in Issue 6, 2015.

Citation: $\mathrm{Ng} \mathrm{L}$, Khan F, Young CA. Symptomatic treatments for amyotrophic lateral sclerosis/motor neuron disease. Cochrane Database of Systematic Reviews 2015, Issue 6. Art. No.: CD011776. DOI: 10.1002/14651858.CD011776.

Copyright (C) 2015 The Cochrane Collaboration. Published by John Wiley \& Sons, Ltd.

\begin{abstract}
A B S T R A C T
This is the protocol for a review and there is no abstract. The objectives are as follows:

To summarise the evidence from Cochrane systematic reviews of all symptomatic treatments for motor neuron disease (MND). If possible, we will compare the effectiveness of agents with similar rationale for use in MND.
\end{abstract}

\section{B A C K G R O U N D}

\section{Description of the condition}

Motor neurone disease (MND), also often known as amyotrophic lateral sclerosis (ALS) is a relatively rare but fatal neurodegenerative disorder of the motor system in adults. It has a reported population incidence of between 1.5 and 2.5 per 100,000 per year worldwide, with the only established risk factors being smoking (Armon 2009), age and family history (Turner 2007). Several known genetic mutations, such as C9ORF72 (Renton 2011), have also been found to cause familial and sporadic amyotrophic lateral sclerosis (MND). The disease occurs throughout adult life, with the peak incidence between 50 to 75 years of age, and is more common in men (in the ratio 3:2) (Turner 2007). Historically, ALS was identified as a clinical syndrome distinguishable from other motor neuron diseases such as primary lateral sclerosis, primary muscular atrophy, and progressive bulbar palsy, based upon the location of first symptom and the extent to which anterior horn cells or corticomotor neurons are initially involved. Typically, loss of motor neurons in the cortex, brain stem, and spinal cord would occur, manifested by upper and lower motor neuron signs and symptoms affecting bulbar, limb, and respiratory muscles. However, it is increasingly evident that ALS is clinically and pathophysiologically diverse, with clear overlap with frontotemporal dementia where there is early loss of frontotemporal system neurons (Turner 2013). Death (usually from respiratory failure) follows on average two to four years after onset, but some people with MND may survive for a decade or more (Forsgren 1983).

Whilst the aetiology of MND is unknown, current evidence suggests that multiple interacting factors contribute to motor neuron injury in MND. The working hypothesis is that MND, like many other chronic diseases, is a complex genetic condition and the relative contribution of individual environmental and genetic factors is likely to be small (Logroscino 2008). The three key pathogenetic hypotheses invoke genetic factors, oxidative stress and glutamater- 
gic toxicity, which result in damage to critical target proteins, such as neurofilaments, and organelles such as mitochondria (Brown 1995; Cookson 1999; Shaw 1997). In addition, there is increasing evidence supporting the hypothesis that altered ribonucleic acid (RNA) processing and aggregation of abnormal proteins play a major role in the pathogenesis of MND (Kim 2013; Verma 2013). The diagnosis of MND is clinical and includes the presence of upper motor neuron and lower motor neuron signs, progression of disease and the absence of an alternative explanation. There is no single diagnostic test at present that can confirm or entirely exclude the diagnosis of MND. Clinicians rely mainly on clinical history and examination, supported by electrodiagnostic studies ( Eisen 2001) and negative findings in neuroimaging and laboratory studies.

The symptoms in MND are diverse and challenging for both patient and diagnostician. They include weakness, spasticity, limitations in mobility and activities of daily living, communication deficits, dysphagia, respiratory compromise, fatigue, sleep disorders, pain and psychosocial distress. The International Classification of Functioning, Health and Disability (ICF) defines a common language for describing the impact of disease at different levels: impairment (body structure and function) and limitation in activity and participation (WHO 2001). Within this framework MND-related impairments (weakness and spasticity), can limit 'activity' or function (mobility, self-care and pain) and 'participation' (driving, employment, family and social reintegration). 'Contextual factors' that may be environmental (extrinsic) or personal (intrinsic) interact with all the other constructs to shape the impact of MND on people with the condition and their families. The burden of disease and economic impact of MND upon patients, their caregivers (often family members) and on society is substantial (Klein 1996). It is estimated that the annual cost impact of MND in the United Kingdom is > $£ 2$ million (NICE 2010). These costs do not include loss of earnings, therapy costs, and formal and informal care, which often make up the bulk of costs but are often not calculated. In Australia, a study of Australian people with MND in the community $(n=44)$ showed that despite most requiring a significant amount of help (more than three times a day) ( $\mathrm{Ng} 2009)$, a quarter of these people received assistance solely from family. It is therefore not surprising that primary caregivers have been estimated to spend a mean of 9.5 hours a day caring for patients, even where there is paid assistance (Chio 2006).

At present, the only approved drug treatment for MND in the United States, Australia and in many European countries is riluzole, which is thought to prolong median survival by about two to three months (Miller 2012). In additional, non-invasive ventilation is also thought to prolong survival (Radunovic 2013). In the absence of a cure or indeed any medical intervention which might stop the progression of MND, the focus is on symptomatic, rehabilitative and palliative therapy with an overall aim of optimising quality of life (QoL).

There are eight reviews in The Cochrane Library that address the effectiveness of a wide range of symptomatic treatment therapies for people with MND (Ashworth 2012; Baldinger 2012; Brettschneider 2013; Dal Bello-Haas 2013; Katzberg 2011; Ng 2011; Radunovic 2013; Young 2011). This overview draws together the findings from these reviews to make the information more accessible.

\section{Description of the interventions}

This review will provide an overview of symptomatic treatments for people with MND. Therefore, reviews of therapies that can alter symptoms but which do not target the processes underlying MND will be included. Therapies will include those that target MND at the impairment level, such as mechanical ventilation for respiratory insufficiency, enteral feeding for maintenance of nutrition impairment, and treatments for spasticity, sialorrhoea, cramps and pain; and those that target MND at the level of activity and participation, such as multidisciplinary care and therapeutic exercise.

\section{How the intervention might work}

A wide range of interventions is used to treat the diverse symptoms and impairments in MND. At the level of impairment, interventions include the following:

- Mechanical ventilation (tracheostomy and non-invasive ventilation) might prolong survival and optimise QoL by supporting ventilation in those with clinically significant respiratory muscle weakness.

- Enteral feeding might improve weight maintenance, survival and QoL by providing a safe and reliable route for nutrition in MND patients who may have a combination of dysphagia, poor appetite and impaired ability to feed themselves leading to reduced oral intake and malnutrition/dehydration.

- Spasticity treatments vary widely and may include physiotherapy (for example therapeutic exercises, stretching, positioning), modalities (for example heat, cold, vibration, electrical stimulation), prescription medication (for example baclofen), non-prescription medication (for example vitamins), chemical neurolysis (botulinum toxin), surgical interventions (for example intrathecal pumps) and alternative therapies (for example reflexology). How the interventions work varies widely from one intervention to another. Most commonly, stretching techniques are used in combination with one or more 'true' muscle relaxants (such as baclofen) (Carter 1998) and such interventions work by lengthening (with or without the assistance of weakening) the agonist muscle.

- Sialorrhoea treatments include suction, drug treatments and more invasive approaches such as injection of botulinum toxin or irradiating the salivary glands, which may improve sialorrhoea and QoL. These interventions work by reducing the 
amount of saliva either through removal (for example, suction) or reduction of salivary output (for example, anticholinergic medications and botulinum toxin injections).

- Cramps - as the origin of cramps is poorly understood, so too are the mechanisms of treatment. Two different pathophysiological mechanisms have been proposed: abnormal excitation of the terminal branches of motor axons (Bertolasi 1993) and hyperexcitability or bistability of motor neurons at a spinal level (Baldissera 1994). Hence, medications most likely work by targeting these mechanisms.

- Pharmacological pain management works by reducing pain. However, analgesics work on different pathways. For example, paracetamol and non-steroidal anti-inflammatory drugs inhibit the production of prostaglandins, which inhibit the production of pain, whilst opiates (such as morphine) imitate natural neuromediators by binding their receptors (such as binding the endorphin receptors).

At the level of activity and participation, interventions include the following:

- Multidisciplinary care might reduce disability and improve quality of life by applying "a problem-solving education process" (Wade 1992), delivered by medical and allied health disciplines (such as physiotherapy, occupational therapy, speech therapy) focused on maximising activity and participation.

- Exercise might reduce disability and fatigue and improve QoL by improving cardiovascular deconditioning and disuse weakness.

\section{Why it is important to do this overview}

As previously mentioned, there are eight reviews in The Cochrane Library that address the effectiveness of a wide range of symptomatic treatment therapies for people with MND specifically (Ashworth 2012; Baldinger 2012; Brettschneider 2013; Dal Bello-Haas 2013; Katzberg 2011; Ng 2011; Radunovic 2013; Young 2011). This overview draws together the findings from multiple Cochrane intervention reviews to give clinicians, policy makers and informed consumers a 'friendly front end' for data from a wide range of reviews. This has the benefit of both making the information more accessible and highlighting the areas that need further research.

\section{O B J E C T I V E S}

To summarise the evidence from Cochrane systematic reviews of all symptomatic treatments for motor neuron disease (MND). If possible, we will compare the effectiveness of agents with similar rationale for use in MND.

\section{METHO D S}

\section{Criteria for considering reviews for inclusion}

\section{Types of reviews}

As per the Cochrane Handbook for Systematic Reviews of Interventions (Becker 2011), we will consider all Cochrane systematic reviews of randomised controlled trials for all treatments for MND. These systematic reviews have the following factors:

- pre-defined objectives

- pre-defined criteria for eligibility of evidence

- an objective systematic search for evidence applying predetermined inclusion and exclusion criteria, and

- explicit and systematic methods for synthesising evidence which attempt to reduce bias.

We will not consider non-systematic reviews for inclusion. Where a systematic review includes both MND and non-MND populations we will include it if the results for people with MND are reported separately.

\section{Types of participants}

We will consider for inclusion reviews of all forms of MND, regardless of clinical pattern (for example bulbar or limb onset). In participants with ALS, the El Escorial and revised El Escorial criteria (Brooks 1994; Brooks 2000) will be used.

\section{Types of interventions}

We will consider for inclusion all non-disease modifying and symptomatic interventions for MND, whether pharmacological or physical. We will not include treatments that target the underlying disease process in MND. These are the subject of a parallel overview of review on disease modifying therapies in MND, which is in development.

\section{Types of outcomes}

In the narrative part of our overview we will report the outcomes reported in the individual Cochrane systematic reviews. Where possible, outcomes will be categorised according to the ICF (WHO 2001), into those that focus on:

- impairment - for example, forced vital capacity (FVC)

- disability or limitation in activity - for example, the revised Amyotrophic Lateral Sclerosis Functional Rating Scale (ALSFRS-R) (the domains of ALSFRS include speech, salivation and swallowing; turning in bed, walking, climbing stairs; dressing and hygiene, handwriting, cutting food; and respiratory insufficiency, dyspnoea, orthopnoea) 
- restriction in participation, and environmental or personal context, or both - for example, patient and caregiver mood, satisfaction with services, social integration

Many of the above scales predate the introduction of the concepts of the ICF domains. The outcome measures cross over the boundaries between the concepts of impairment, disability and participation. For example, ALSFRS lists impairments such as dyspnoea and orthopnoea, as well as disabilities like walking or climbing stairs. Other important outcomes such as survival and $\mathrm{QoL}$ are not strictly covered by the ICF concepts (although there is again cross over of boundaries between ICF concepts and these outcomes). Nevertheless, the ICF provides an important framework, which allows the use of a common standardised language worldwide, hence its application in this overview. Scales used must have been validated as having good reproducibility, face validity and correlation with other scales measuring the same attribute.

Outcome time points will be divided into short-term (up to 3 months), medium-term (3 to 12 months) and long-term (at least 12 months) for both primary and secondary outcomes.

\section{Primary outcomes}

Primary outcomes will focus on domains within QoL and health status after 12 months, such as the Short Form-36 (SF-36) or Visual Analogue Scale (VAS) on life satisfaction and well-being. As quality of life is a broad concept, it is likely that different scales measuring various aspects of quality of life could be used.

\section{Secondary outcomes}

These will include the following short-term, medium-term and long-term outcomes:

- outcomes that relate to impairment - for example, forced vital capacity (FVC)

- outcomes that relate to disability or limitation in activity for example, Amyotrophic Lateral Sclerosis Severity Scale (ALSSS) and ALSFRS

- outcomes that relate to restriction in participation, and environmental or personal context, or both - for example, Caregiver Strain Index (CSI), Utrecht Coping List (UCL)

- survival

- hospitalisation such as readmissions and hospital length of stay (LOS)

- cost-effectiveness of care

We will also report adverse events that may have resulted from the intervention. We will define serious adverse effects as those events that were fatal, life-threatening or required prolonged hospitalisation.

\section{Search methods for identification of reviews}

We will search the Cochrane Database of Systematic Reviews (CDSR) and the Database of Abstracts of Reviews of Effects (DARE) (current issues in The Cochrane Library), MEDLINE (January 1966 to current), EMBASE (January 1980 to current) and CINAHL Plus (January 1937 to current issue) for systematic reviews of MND.

The search strategy for MEDLINE is shown in Appendix 1.

\section{Data collection and analysis}

\section{Selection of reviews}

Two overview authors will select systematic reviews for inclusion and will resolve disagreements by consensus following discussion with a third author.

\section{Data extraction and management}

Two overview authors will independently collect data from published systematic reviews with a data collection form designed specifically to include all the data needed. We will use 'Characteristics of included reviews' tables to present the essential features of the included reviews.

We will resolve disagreements by consensus following discussion with a third author.

We will contact the review authors or extract data from the relevant trials if further information is required.

We will extract the following characteristics from the reviews:

- date assessed as up to date

- objectives

- participants

- interventions

- comparisons

- outcomes in the review for which data are available

- limitations

\section{Assessment of methodological quality of included reviews}

Two overview authors, who are not authors of the original included reviews, will independently assess the methodological quality of each review included in the overview. For this purpose we will use the assessment of multiple systematic reviews (AMSTAR) tool developed by Shea 2007, which has acceptable inter-rater agreement, construct validity and feasibility (Shea 2009). Two overview authors will also independently assess the quality of the evidence in the included reviews with the Grading of Recommendations Assessment, Development and Evaluation (GRADE) approach (Guyatt 2008). In both cases, disagreements will be resolved by discussion, if necessary with a third author. 


\section{Data synthesis}

We will present data predominantly via a narrative review. We will report the evidence for each intervention from each review and its strength in an 'Overview of reviews' table using the GRADE approach. We will use the following criteria to assess the quality of the evidence: study limitations (that is, risk of bias), consistency of effect, imprecision, indirectness and publication bias. We will assess the evidence using GRADE criteria where reviews lack 'Summary of findings' tables. Data will be presented by symptom. In 'Overview of reviews' tables we will present:

- beneficial and harmful outcomes

- frequency or severity of these outcomes in the control groups

- estimates of the relative and absolute effects of the interventions

- indications of the risk of bias (which may vary by outcome and comparison)

- comments

Where more than one eligible review of a particular intervention is found and the conclusions agree we will report this; where the conclusions differ, we will further explore this, taking into account the AMSTAR scores of the included reviews.

Formal multiple-treatments meta-analysis may be appropriate if the same outcome has been used. To be considered comparable, the outcomes should have been measured in clinically equivalent ways over clinically similar periods of follow-up. However, given the diverse nature of the symptoms and impairments, direct or indirect comparisons of the interventions are likely to be more appropriate for inclusion in an intervention review rather than in this overview.

\section{ACKNOWLEDGEMENTS}

We thank the Cochrane Neuromuscular Disease (NMD) Group for their assistance.

Editorial support from the Cochrane NMD Group for this overview is funded by the Motor Neuron Disease Association.

This project was supported by the National Institute for Health Research via Cochrane Infrastructure funding to the Cochrane Neuromuscular Disease Group. The views and opinions expressed therein are those of the authors and do not necessarily reflect those of the Systematic Reviews Programme, NIHR, NHS or the Department of Health. The editorial base of the Cochrane NMD Group also receives support from the Medical Research Council (MRC) Centre for Neuromuscular Diseases.

For some parts of the methods section of this overview protocol we used Hughes 2013 as a template.

\section{R E F E R E N C E S}

\section{Additional references}

\section{Armon 2009}

Armon C. Smoking may be considered an established risk factor for sporadic ALS. Neurology 2009;73(20):1693-8.

\section{Ashworth 2012}

Ashworth NL, Satkunam LE, Deforge D. Treatment for spasticity in amyotrophic lateral sclerosis/motor neuron disease. Cochrane Database of Systematic Reviews 2012, Issue 2. [DOI: 10.1002/14651858.CD004156.pub4]

\section{Baldinger 2012}

Baldinger R, Katzberg HD, Weber M. Treatment for cramps in amyotrophic lateral sclerosis/motor neuron disease. Cochrane Database of Systematic Reviews 2012, Issue 4. [DOI: 10.1002/14651858.CD004157.pub2]

\section{Baldissera 1994}

Baldissera F, Cavallari P, Dworzak F. Motor neuron 'bistability'. A pathogenetic mechanism for cramps and myokymia. Brain 1994;117(Pt 5):929-39.

Becker 2011

Becker LA, Oxman AD. Chapter 22: Overview of reviews. In: Higgins JPT, Green S editor(s). Cochrane Handbook for Systematic Reviews of Interventions Version 5.1.0 (updated
March 2011). Available from www.cochrane-handbook.org:

The Cochrane Collaboration, 2011.

Bertolasi 1993

Bertolasi L, De Grandis D, Bongiovanni LG, Zanette GP, Gasperini $M$. The influence of muscular lengthening on cramps. Annals of Neurology 1993;33(2):176-80.

Brettschneider 2013

Brettschneider J, Kurent J, Ludolph A. Drug therapy for pain in amyotrophic lateral sclerosis or motor neuron disease. Cochrane Database of Systematic Reviews 2013, Issue 6. [DOI: 10.1002/14651858.CD005226.pub3]

Brooks 1994

Brooks BR. Escorial World Federation of Neurology criteria for the diagnosis of amyotrophic lateral sclerosis. Subcommittee on Motor Neuron Diseases/Amyotrophic Lateral Sclerosis of the World Federation of Neurology Research Group on Neuromuscular Diseases and the El Escorial "Clinical limits of amyotrophic lateral sclerosis" workshop contributors. Journal of the Neurological Sciences 1994;124(Suppl):96-107.

\section{Brooks 2000}

Brooks BR, Miller RG, Swash M, Munsat TL, World Federation of Neurology Research Group on Motor Neuron Diseases. El Escorial revisited: revised criteria for the 
diagnosis of amyotrophic lateral sclerosis. Amyotrophic Lateral Sclerosis and Other Motor Neuron Disorders 2000;1 (5):293-9.

\section{Brown 1995}

Brown RH Jr. Amyotrophic lateral sclerosis: recent insights from genetics and transgenic mice. Cell 1995;80(5): 687-92.

Carter 1998

Carter GT, Miller RG. Comprehensive management of amyotrophic lateral sclerosis. Physical Medicine and Rehabilitation Clinics of North America 1998;9(1):271-84, viii-ix.

Chio 2006

Chio A, Gauthier A, Vignola A, Calvo A, Ghiglione P, Cavallo E, et al. Caregiver time use in ALS. Neurology 2006; 67(5):902-4.

Cookson 1999

Cookson MR, Shaw PJ. Oxidative stress and motor neurone disease. Brain Pathology 1999;9(1):165-86.

\section{Dal Bello-Haas 2013}

Dal Bello-Haas V, Florence JM. Therapeutic exercise for people with amyotrophic lateral sclerosis or motor neuron disease. Cochrane Database of Systematic Reviews 2013, Issue 5. [DOI: 10.1002/14651858.CD005229.pub3]

Eisen 2001

Eisen A, Swash M. Clinical neurophysiology of ALS. Clinical Neurophysiology 2001;112(12):2190-201.

\section{Forsgren 1983}

Forsgren L, Almay BG, Holmgren G, Wall S. Epidemiology of motor neuron disease in northern Sweden. Acta Neurologica Scandinavica 1983;68(1):20-9.

\section{Guyatt 2008}

Guyatt GH, Oxman AD, Vist GE, Kunz R, Falck-Ytter Y, Alonso-Coello P, et al. GRADE: an emerging consensus on rating quality of evidence and strength of recommendations. BMJ 2008;336(7650):924-6.

\section{Hughes 2013}

Hughes RAC, Lunn MPT, Frost C, van Schaik IN. Treatments for chronic inflammatory demyelinating polyradiculoneuropathy (CIDP): an overview of systematic reviews. Cochrane Database of Systematic Reviews 2013, Issue 2. [DOI: 10.1002/14651858.CD010369]

\section{Katzberg 2011}

Katzberg HD, Benatar M. Enteral tube feeding for amyotrophic lateral sclerosis/motor neuron disease. Cochrane Database of Systematic Reviews 2011, Issue 1. [DOI: 10.1002/14651858.CD004030.pub3]

Kim 2013

Kim HJ, Kim NC, Wang YD, Scarborough EA, Moore J, Diaz Z, et al. Mutations in prion-like domains in hnRNPA2B1 and hnRNPA1 cause multisystem proteinopathy and ALS. Nature 2013;495(7442):467-73.

Klein 1996

Klein LM, Forshew DA. The economic impact of ALS. Neurology 1996;47(4 Suppl 2):S126-9.

\section{Logroscino 2008}

Logroscino G, Traynor BJ, Hardiman O, Chio A, Couratier P, Mitchell JD, et al. EURALS. Descriptive epidemiology of amyotrophic lateral sclerosis: new evidence and unsolved issues. Journal of Neurology, Neurosurgery \& Psychiatry 2008; 79(1):6-11.

\section{Miller 2012}

Miller RG, Mitchell JD, Moore DH. Riluzole for amyotrophic lateral sclerosis (ALS)/motor neuron disease (MND). Cochrane Database of Systematic Reviews 2012, Issue 3. [DOI: 10.1002/14651858.CD001447.pub3]

\section{Ng 2009}

$\mathrm{Ng}$ L, Khan F. Multidisciplinary care for adults with amyotrophic lateral sclerosis or motor neuron disease. Cochrane Database of Systematic Reviews 2009, Issue 4. [DOI: 10.1002/14651858.CD007425.pub2]

\section{Ng 2011}

Ng L, Talman P, Khan F. Motor neurone disease: disability profile and service needs in an Australian cohort. International Journal of Rehabilitation Research 2011;34(2): $151-9$.

\section{NICE 2010}

National Institute for Health and Clinical Excellence. National costing report: Non-invasive ventilation for MND (July 2010). http://www.nice.org.uk/guidance/cg105/ resources/motor-neurone-disease-noninvasive-ventilationcosting-report2 2010.

\section{Radunovic 2013}

Radunovic A, Annane D, Rafiq MK, Mustfa N. Mechanical ventilation for amyotrophic lateral sclerosis/motor neuron disease. Cochrane Database of Systematic Reviews 2013, Issue 3. [DOI: $10.1002 / 14651858 . C D 004427 . p u b 3]$

\section{Renton 2011}

Renton AE, Majounie E, Waite A, Simón-Sánchez A, Rollinson S, Gibbs JR, et al. A hexanucleotide repeat expansion in C9ORF72 Is the cause of chromosome 9p21linked ALS-FTD. Neuron 2011;72(2):257-68.

\section{Shaw 1997}

Shaw PJ, Ince PG. Glutamate, excitotoxicity and amyotrophic lateral sclerosis. Journal of Neurology 1997;244 (Suppl 2):S3-14.

Shea 2007

Shea BJ, Grimshaw JM, Wells GA, Boers M, Andersson N, Hamel C, et al. Development of AMSTAR: a measurement tool to assess the methodological quality of systematic reviews. BMC Medical Research Methodology 2007;7:10.

Shea 2009

Shea BJ, Hamel C, Wells GA, Bouter LM, Kristjansson E, Grimshaw J, et al. AMSTAR is a reliable and valid measurement tool to assess the methodological quality of systematic reviews. Journal of Clinical Epidemiology 2009; 62(10):1013-20.

\section{Turner 2007}

Turner MR, Al-Chalabi A. Clinical phenotypes. In: Kiernan M editor(s). The Motor Neurone Disease Handbook. 
Pyrmont: Australasian Medical Publishing Company

Limited, 2007:56-73.

\section{Turner 2013}

Turner MR, Hardiman O, Benatar M, Brooks BR, Chio

A, de Carvalho $\mathrm{M}$, et al. Controversies and priorities in

amyotrophic lateral sclerosis. Lancet Neurology 2013;12(3):

310-22.

\section{Verma 2013}

Verma A, Tandan R. RNA quality control and protein aggregates in amyotrophic lateral sclerosis: a review. Muscle \& Nerve 2013;47(3):330-8.

\section{Wade 1992}

Wade DT. Measurement in Neurology Rehabilitation.

Oxford: Oxford University Press, 1992.

\section{WHO 2001}

World Health Organization. International Classification of Functioning, Disability, and Health (ICF). International Classification of Functioning, Disability, and Health (ICF). Geneva: World Health Organization, 2001.

\section{Young 2011}

Young CA, Ellis C, Johnson J, Sathasivam S, Pih N. Treatment for sialorrhea (excessive saliva) in people with motor neuron disease/amyotrophic lateral sclerosis. Cochrane Database of Systematic Reviews 2011, Issue 5. [DOI: 10.1002/14651858.CD006981.pub2]

* Indicates the major publication for the study

\section{A P P E N D I C ES}

\section{Appendix I. MEDLINE (OvidSP) search strategy}

Database: Ovid MEDLINE(R)

Search Strategy:

1 exp Motor Neuron Disease/ (19713)

2 (moto $\$ 1$ neuron $\$ 1$ disease $\$ 1$ or moto?neuron $\$ 1$ disease $\$ 1) . m p$. (6410)

3 ((Lou Gehrig\$1 adj5 syndrome\$1) or (Lou Gehrig\$1 adj5 disease)).mp. (89)

4 charcot disease.tw. (12)

5 Amyotrophic Lateral Sclerosis.mp. (16281)

6 or/1-5 (23880)

7 meta-analysis/ (47945)

8 meta-analysis.pt. (47945)

9 (meta analy\$ or metaanaly\$ or meta?analy\$).tw. (56324)

10 ((health technology adj5 assessment) or hta).tw. (2310)

11 (systematic adj3 review\$).mp. (48234)

12 (systematic adj3 overview\$).mp. (675)

13 consensus development conference.pt. (9061)

14 practice guideline.pt. (19018)

15 or/7-14 (128407)

166 and 15 (229)

17 remove duplicates from 16 (205) 


\section{CONTRIBUTIONSOFAUTHORS}

LN prepared the first draft of the protocol. FK and CY commented on and edited the first and subsequent drafts, and approved the final version.

\section{DECLARATIONSOF INTEREST}

LN and FK: no known conflicts of interest. LN, FK and CY were authors on reviews likely to be included in this overview.

CY has published and lectured on fatigue in various neurological conditions, including MND, and is very involved in MND research, for which the NHS receives payment. She has had funding from pharmaceutical companies to attend and present at educational meetings.

\section{SOURCES OF SUPPORT}

\section{Internal sources}

- Department of Rehabilitation Medicine, Royal Melbourne Hospital, Australia.

\section{External sources}

- No sources of support supplied 


\section{University Library}

\section{- M M I N E R VA A gateway to Melbourne's research publications}

Minerva Access is the Institutional Repository of The University of Melbourne

Author/s:

Ng, L;Khan, F;Young, CA;Galea, M

Title:

Symptomatic treatments for amyotrophic lateral sclerosis/motor neuron disease

Date:

2017-01-01

Citation:

Ng, L., Khan, F., Young, C. A. \& Galea, M. (2017). Symptomatic treatments for amyotrophic lateral sclerosis/motor neuron disease. COCHRANE DATABASE OF SYSTEMATIC REVIEWS, 1 (1), https://doi.org/10.1002/14651858.CD011776.pub2.

Persistent Link:

http://hdl.handle.net/11343/118540 